Kompass

Onkologie

Kompass Onkol 2020;7:210-216

DOI: 10.1159/000513212

\title{
Digitalisierung in der Medizin - nicht «ob» sondern «wie»!
}

Nie zuvor wurde die digitale Transformation des Gesundheitswesens von allen Berufsgruppen derart vorangetrieben wie in den letzten Monaten. Wo es möglich war, wurden persönliche Arzt-PatientenKontakten vermieden und auf digitale Alternativen gesetzt. Ängste wurden geradezu weggespült und neue Techniken, aber auch ein neuer Umgang mit den Patienten ausgetestet. Ein Handlungsmuster, was die jungen Ärztinnen und Ärzte als «digital natives» beherrschen. Dennoch benötigt es auch eine Weiterentwicklung der Aus- und Weiterbildung von Ärzten, um den wachsenden Ansprüchen der Patienten und neuen Techniken wie Big Data, Augmented Reality (AR) und künstlicher Intelligenz (KI) gerecht zu werden.

\section{Telemedizin}

Die Telemedizin hat in den vergangenen Jahren zum Beispiel mit mehreren Anbietern in der Dermatologie Einzug gehalten. Unterschiedliche Lösungen werden angeboten, mit Videokonsultationen als synchrones Verfahren oder mittels asynchroner «Store-andForward» Technologie. Bei Letzterem werden die Bilder des Patienten räumlich wie zeitlich getrennt beurteilt.

Eine lokale Verknüpfung mit der Hautarztpraxis, wo im Anschluss an die teledermatologische Beurteilung ein konkreter physischer Vorstellungstermin vereinbart werden kann, bietet etwa OnlineDoctor. Weitere Lösungen wie derma2go oder dermanostic setzen jeweils auf ein Expertenteam, die Auswahl des Arztes durch den Patienten ist jedoch nicht möglich.

\section{Digitale Gesundheitsanwendungen}

Kurz bevor steht die Zulassung von Apps auf Rezept, den sogenannten Digitalen Gesundheitsanwendungen (DiGA), die Fortschritte unter anderem in der Betreuung von Patienten mit chronischen Dermatosen bringen werden. Vereinzelt sind diese auch bereits im App- und Google Play Store erhältlich.

In der Umfrage der BARMER, welche sich an 1000 Haus- und Fachärzte richtete, zeigte sich eine überwiegende Zustimmung der befragten Ärzteschaft hinsichtlich DIGAs. Es wünschen sich 74\% der befragten Ärzte einen Überblick über das App Angebot und 61\% Informationen über den Nutzen von Apps für die Patienten. Die Ärztekammern und Kassenärztlichen Vereinigungen werden als wünschenswerte Informationsquellen angesehen.

\section{Weitere Anwendungsmöglichkeiten}

Die Vernetzung des Gesundheitswesens, gerade auch die Kommunikation über die Sektoren hinweg, muss verbessert werden. Wieso ist bis heute kein einheitlicher Messenger-Dienst für Mitarbeiter im Gesundheitswesen zur Kommunikation auch mit Patientendaten etabliert? Warum werden bürokratische Prozesse nicht digitalisiert und automatisiert? Warum sind FAX und Brief weiterhin unsere HauptKommunikationsinstrumente? Wieso wird Al nicht verstärkt für die Patientenedukation genutzt?
Dies sind nur einige der Fragen, die digital affinen, jungen Ärztinnen und Ärzten häufig durch den Kopf gehen. Den entsprechenden Gremien im Gesundheitswesen fehlt diese digitale Affinität aber oft. Der Bezug zu praktischen Anwendern ist unerlässlich. Hier ist der generationsübergreifende Informationsaustausch wichtig und muss dringend durch Einbindung von jungen Ärztinnen und Ärzten etabliert werden.

Es braucht also zeitnah digital affine Ärztinnen und Ärzte in den Gremien, aber genauso in der alltäglichen Versorgung, die den Anfragen der Patienten gerecht werden können!

\section{Studium}

Der Wissensgrundstein späteren Arbeitens wird in der Ausbildung von Ärztinnen und Ärzten, also im Studium, gelegt. Wir benötigen im Studium in Deutschland eine schnelle, umfängliche und praxisnahe Einführung digitaler Kenntnisse. Hierzu zählen die technologischen Basics wie Sprache und Aufbau der Informationstechnologie (IT), Begriffskenntnisse, aber auch anwendungsbezogenes Wissen, welches vor allem bei DIGAs und der Anwendung der elektronischen Patientenakte (ePA) zum Einsatz kommen wird. Erste Fortschritte wurden in der Einführung mit Wahlfächern gemacht. Es ist nun erforderlich, diesen Prozess zu beschleunigen und zusätzlich ärztliches Lehrpersonal aus der Praxis einzubinden.

\section{Weiterbildung}

Digitale Kompetenzen sind bisweilen in der Weiterbildungsordnung (MWBO) für Ärzte in Weiterbildung noch nicht abgebildet. Auch in der neuen MWBO, welche aktuell umgesetzt wird, sind digitale Kenntnisse auch nicht vorgesehen. Es obliegt also aktuell noch jedem Weiterbildenden und auch jedem Weiterzubildenden sich mit Grundkenntnissen der IT und Datenanalyse, aber auch der Anwendung neuer Tech-

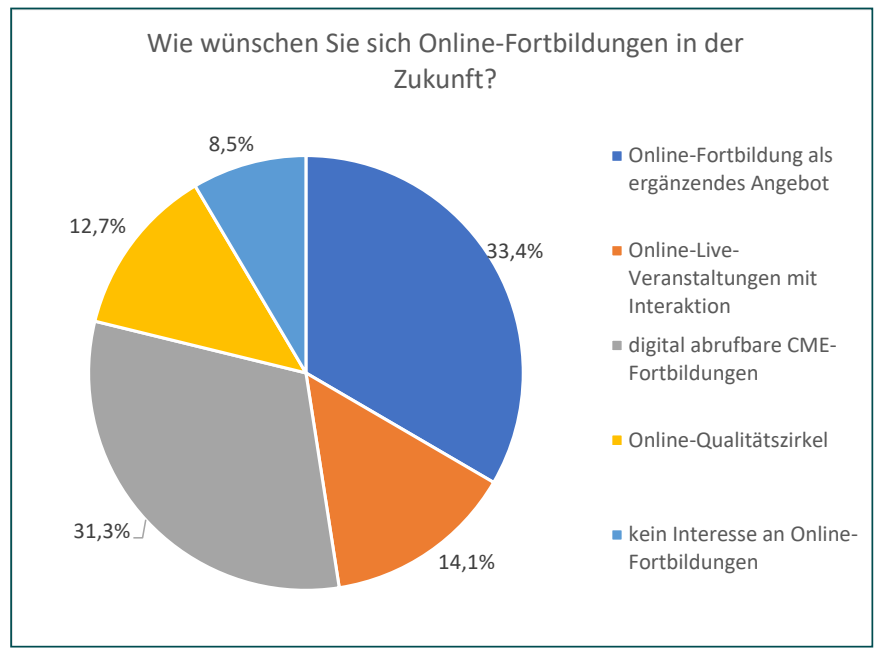

In einer Umfrage des Berufsverbandes der Deutschen Dermatologen unter knapp 530 Teilnehmern zeigt sich ein großes Interesse an Online-Fortbildungsmöglichkeiten. 
niken, vertraut zu machen. Erste Impulse werden aktuell auch durch die Bundesärztekammer (BÄK) mit dem (Muster-)Kursbuch zur Zusatzweiterbildung Medizinische Informatik gesetzt. Inwieweit diese und andere Zusatzqualifikationen angenommen werden, bleibt abzuwarten. Für die Zukunft wünschen sich junge Ärztinnen und Ärzte in der Weiterbildung eine grundlegende Kenntnis über digitale Technologien und eine optimale technische Ausstattung am jeweiligen Arbeitsplatz. Dies würde einen weiteren Grundstein für die Transformation und Zukunftssicherheit des deutschen Gesundheitswesens bieten.

\section{Arzt für digitale Medizin}

Auch wenn die Implementation digitaler Bildung in Aus- und Weiterbildung von Ärztinnen und Ärzten noch einige Jahre in Anspruch nehmen wird, braucht es bereits jetzt Lösungen für den «digitalen» Patienten, der innovative Tools bereits heute nutzt. Gerade durch DIGAs und deren Verordnungsmöglichkeit durch die Krankenkassen bei passenden Diagnosen, werden Patienten den Digitalisierungsschub im Gesundheitswesen erleben können. Bei Rückfragen sollten diese Patienten geeignete Ärzte finden können, die sich neuen Technologien nicht verschließen. Daher hat das Bündnis Junge Ärzte, ein Zusammenschluss von 24 jungen Berufsverbänden und Fachgesellschaften, bereits am 15. Juni 2020 als Übergangslösung einen «Arzt für digitale Medizin» gefordert.

Dass es keinen alleinigen Facharzt für Telemedizin oder digitale Medizin geben kann, hat die Bundesärztekammer (BÄK) bereits 2014 klargestellt. Vielmehr kann die Forderung als Aufruf zur Implementation eines Qualitätssigels oder Registers verstanden werden. Di- gital affinen Patienten muss eine Orientierung gegeben werden, um Enttäuschungen und Unverständnis auf Seiten von Patienten, aber auch von Ärzten, zu vermeiden.

Auf Seiten der Ärzteschaft benötigt es zeitnah qualitative Fortbildungsangebote über die verschiedenen, neuen, digitalen Tools, die in einer gesonderten Qualifikation, dem Arzt für digitale Medizin, münden könnten.

\section{Fazit}

An der digitalen Transformation des Gesundheitswesens führt also kein Weg vorbei. Die Corona-Pandemie kann als digitaler Evolutionsbeschleuniger wahrgenommen werden. Für die Zukunft müssen aber auch die Grundlagen in Aus- und Weiterbildung geschaffen werden. Ärztinnen und Ärzte stehen der digitalen Transformation offenen gegenüber und müssen proaktiv in die Ausgestaltung der Prozesse eingreifen und eingebunden werden. Nur dann können digitale Innovationen ein Erfolg zum Wohle des Patienten werden.

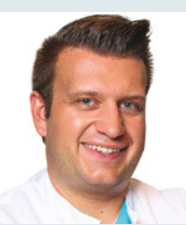

Kontaktadresse: Max Tischler, Sprecher Bündnis Junge Ärzte, Junge Dermatologen (JuDerm) im Berufsverband der Deutschen Dermatologen (BVDD), Stv. Vorsitzender Arbeitskreis Junge Ärzte der Ärztekammer

Westfalen-Lippe; Hautärzte am Markt Dortmund, Markt 4,44137

Dortmund, max.tischler@buendnisjungeaerzte.org

\section{Barcamp Hautkrebs}

\section{Das Format, das Partizipation statt Langeweile bietet}

In den Bereichen Bildung und Gesundheit verändert sich durch die Digitalisierung vieles. Informelles, selbstgesteuertes und vernetztes Lernen ist bei den Patienten längst angekommen. Sie wollen mehr wissen, sind gut vernetzt und bilden sich digital weiter. Das traditionelle Laien-Experten-Verhältnis verändert sich zunehmend und auch der Austausch über die eigene Krankheit findet nicht mehr isoliert zwischen Arzt und Patient statt, sondern in diesen Netzwerken [1]. Im Januar 2020 fand das erste Barcamp Hautkrebs in Bad Soden in der Nähe von Frankfurt statt. Die zentrale Frage war: «Was bewegt Menschen mit Hautkrebs?»

Christiane Weber, Astrid Doppler und Katharina Kaminski (beide Melanom Info Deutschland e.V.) [2] initiierten das erste Barcamp für onkologische Patienten mit dem Ziel, eine moderne Veran-

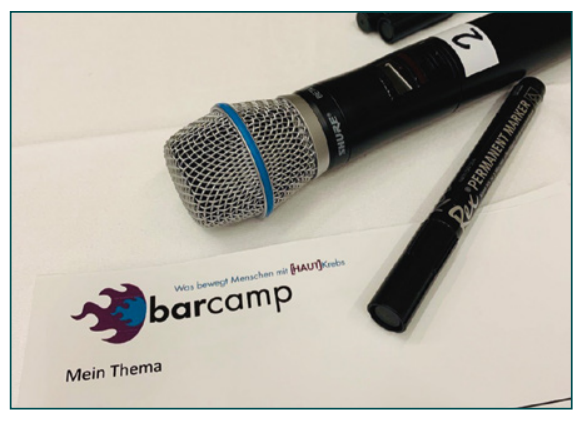

«Mein Thema» - bei einem Barcamp können alle Teilnehmenden ihr eigenes Thema ins Programm bringen. staltung mit Patienten durchzuführen und kein fertiges Vortragsprogramm für Patienten vorzugeben.

Die Idee war bei der 5. Nationalen Versorgungskonferenz Hautkrebs (NVKH) im März 2019 entstanden. Den Organisatorinnen war es wichtig, dass die Teilnehmenden sich mit ihrem individuellen Wissen einbringen können. Unterstützt wurde diese Initiative durch die Arbeitsgruppe «Patientenorientierung» der NVKH, die Arbeitsgemeinschaft Dermatologische Onkologie (ADO) sowie verschiedene Patientenorganisationen, wie das Hautkrebsnetzwerk Deutschland (HKND) oder Melanoma Patient Network Europe (MPNE).

\section{Was ist ein Barcamp?}

Ein Barcamp ist ein Veranstaltungsformat, das auch «Un-Konferenz» oder «Mitmach-Konferenz» genannt wird, weil Programm und Referenten nicht vorab feststehen. Teilnehmen kann jeder, niemand wird explizit eingeladen, es gibt keine eingeladenen Redner*innen. Jeder kann sich einbringen, mitdiskutieren, zuhören und mitgestalten.

Zu Beginn des Barcamps gibt es eine Einführung in das Vorgehen, anschließend werden die Themen vorgestellt. Ausgangspunkt kann ein Input (zum Beispiel ein Kurzvortrag), eine Frage oder ein Diskussionsbedarf sein - ganz am Interesse der Teilnehmenden orientiert. Interessierte können etwas vorbereiten oder spontan ein Thema einbringen. Die Teilnehmenden, hier auch Teilgebende genannt, stehen im Mittelpunkt. 


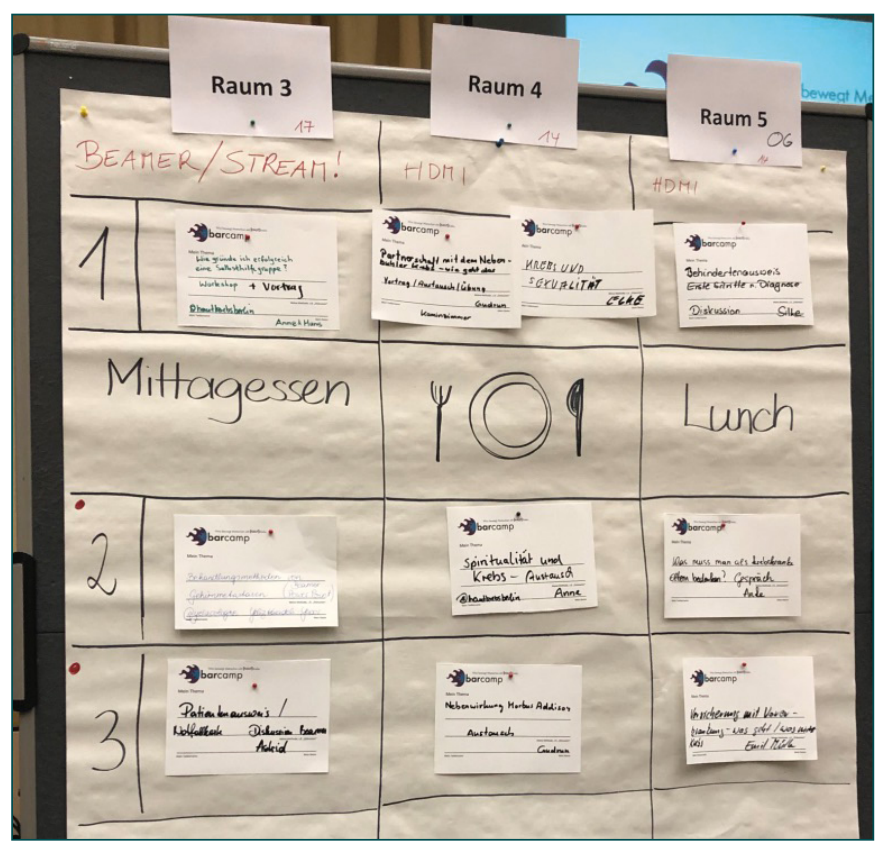

Planung des Programms beim 1. Barcamp Hautkrebs (Sessionplanung) mit allen Teilnehmern.

Ohne hierarchischen Unterschieden (Referent/Zuhörer) sind alle gleichberechtigt. Eine offene und wertschätzende Kommunikation während der Veranstaltung ergibt sich hieraus. Die Workshops werden auf einem Barcamp «Sessions» genannt und dauern 45 Minuten. Es gibt ausreichend Zeitslots und Räume, sodass viele Programmpunkte parallel stattfinden können. Alle, die ein Thema vorschlagen, können dies im Rahmen eigener Workshops vorstellen oder diskutieren. Wie bei einem «normalen» Workshop-Programm können sich Teilnehmende vor Ort entscheiden, ob und in welcher Weise sie sich engagieren, selber aktiv werden und an welchen Workshops sie teilnehmen.

Der Begriff Barcamp kommt aus der IT-Branche. «Camp» steht für das offene Zusammenkommen und verdeutlicht den Freiraum für die Teilnehmer, miteinander etwas zu gestalten. «Bar» steht für das Leerzeichen und versinnbildlicht den leeren Rahmen, den ein Barcamp den Teilnehmenden gibt.

Barcamps eignen sich zum Wissens- und Erfahrungsaustausch, um Ziele in einem Dialog zu erarbeiten oder um die Zusammenarbeit über Grenzen hinweg zu vereinfachen. Zu diesen Grenzen gehören z.B. intersektorale Grenzen zwischen Abteilungen, Standorten oder unterschiedlichen Seiten in einem System. Im Sinne von Patient Empowerment liegen die Vorteile im medizinischen Sektor vor allem in diesen 3 genannten Einsatzbereichen. Mittlerweile haben sich auch digitale Barcamps bewährt.

\section{Online Community}

Das Event selbst fand als Präsenzveranstaltung statt, Vor- und Nachbereitung waren mehrheitlich digital. Im Vorfeld wurde eine Social-MediaStrategie erarbeitet und ab Oktober 2019 eine Community in den Sozialen Medien aufgebaut. Für das Barcamp Hautkrebs gibt es neben der Webseite einen Account bei Twitter, Instagram und Facebook. Dort konnten regelmäßig unterstützende Patientenorganisationen, andere aktive Gruppen und Institutionen, sowie die Teilnehmenden direkt erreicht werden. In regelmäßigen Posts, live Online FAQ-Sessions und kur-

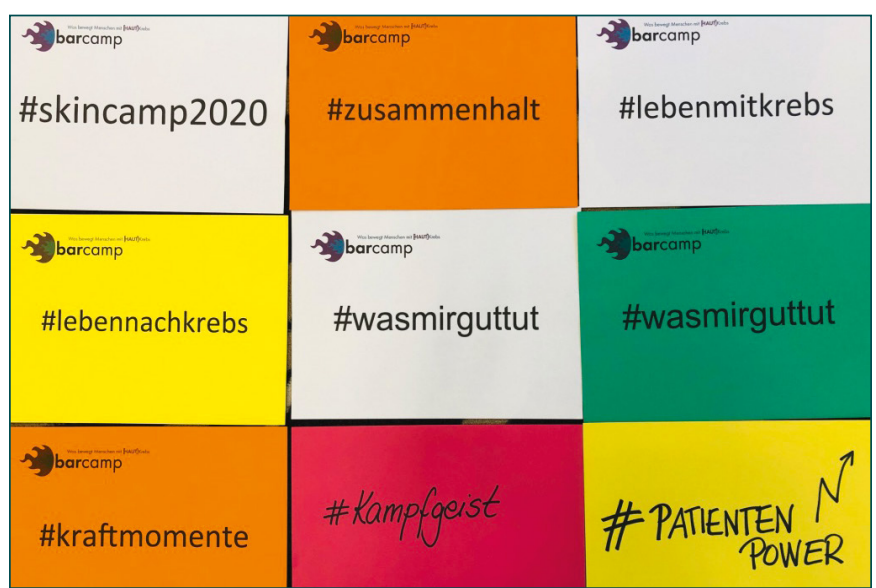

Mit vielen Hashtags wurde über das Barcamp in den Sozialen Medien berichtet.

\section{Barcamp Hautkrebs}

Nähere Informationen findet man auf der Webseite www. barcamp-hautkrebs.de, den Social Media Kanälen bei Twitter @skincamp2020, Instagram @skin.camp und Facebook. \#skincamp2020 bzw. \#skincamp2021

zen Videos zum Ablauf wurden diese über mehrere Wochen an das Format herangeführt. Die häufige Interaktionsmöglichkeit hat gezeigt, dass eine erwartete Hemmschwelle auf dem Weg abgebaut werden konnte. Ebenso konnte dadurch über einen längeren Zeitraum Aufmerksamkeit für das Thema Hautkrebs und die Themen der Betroffenen geschaffen werden.

\section{Aktive Beteiligung}

Über 100 Betroffene, Angehörige, aktive Krebsblogger sowie Gesundheitswissenschaftler haben teilgenommen. In einem einstündigen moderierten Planungstreffen zu Beginn konnten 25 Sessions festgelegt werden. Die Themen reichten von Hautkrebsscreening über Therapiemöglichkeiten bis zu psychosozialen Aspekten (Notfallausweis, Versicherungen). Zwei Sessions (Austausch unter Angehörigen und Austausch unter Betroffenen) wurden als Walk\&Talk im nahegelegenen Park angeboten.

«Informieren im Internet» war eine der bestbesuchten Sessions. Fazit: Die Teilnehmer wünschen sich einen leichteren Zugang zu Informationen aus seriösen, neutralen Quellen (Qualitätssiegel). Die Informationen sollten in erster Linie digital und interaktiv aufgearbeitet sein, z.B. in Form von Online Seminaren, Interviews oder Podcasts. Nach eigenen Angaben befürworten die Patienten den Einsatz von digitalen Medien mehr als ihre Behandler*innen.

Um denjenigen, die krankheitsbedingt oder aus anderen Gründen nicht reisen konnten, ebenfalls den Austausch zu ermöglichen, wurden 2 Sessions im Livestream in die Online-Gruppe Hautkrebs übertragen. So bestand die Möglichkeit, sich aktiv über einen Chat zu beteiligen. Das Angebot wurde sehr gut angenommen.

\section{Zusammenfassung und Ausblick}

Beim Barcamp gab es mehrere Anknüpfungspunkte, welche im Rahmen der Digitalisierung in der Patientenkommunikation auf- 


\section{Melanom Info Deutschland e.V. - Online Community Hautkrebs}

Melanom Info Deutschland - MID e.V. wurde 2017 von Astrid Doppler und Katharina Kaminski gegründet. Fokus ist der online basierte Patientenaustausch. «Unser Ziel ist es, dass möglichst viele Hautkrebsbetroffene ihre Erkrankung überleben und das bei möglichst guter Lebensqualität. Mit jedem Patienten fließt neues Patientenwissen in unsere Community ein und erweitert unseren Erfahrungsschatz. Wir strukturieren und koordinieren dieses Netzwerk, und vertreten unsere Interessen nach außen gegenüber Fachgesellschaften, Politik und allen anderen Akteuren im Gesundheitswesen.» Die Gruppe richtet sich an alle Hautkrebspatienten.

Webseite: www.melanominfo.com

\section{Infoportal Hautkrebs}

Übergeordnete Ziele des Nationale Versorgungskonferenz Hautkrebs (NVKH) e.V. sind Prävention von Hautkrebs sowie die umfangreiche Versorgung von Menschen mit Hautkrebs. Zu diesem Zweck baut der NVKH e.V. ein umfassendes Informations- \& Netzwerkportal auf, welches zu Hautkrebs, aber auch zu angrenzenden Themen informiert. Es wird Interessierten und Betroffenen nicht nur einen Überblick zu den einzelnen Hautkrebsformen verschaffen, sondern auch die Möglichkeit bieten, sich tiefgründiger mit den einzelnen Aspekten der Tumorerkrankung auseinanderzusetzen.

Webseite: www.nvkh.de gegriffen werden sollten. Die Ergebnisse der Konferenz fanden bereits eine Fortsetzung in einzelnen Projekten.

So wurde beispielsweise die Idee eines Podcasts für Hautkrebspatienten von der ADO bereits im April umgesetzt. Darüber hinaus arbeitet die AG «Patientenorientierung» der NVKH am Infoportal Hautkrebs einer neutralen, unabhängigen Informationsplattform [3]. Die Webseite soll im Februar 2021 zur 7. NVKH gelauncht werden. Aus dieser Arbeitsgruppe sind bereits mehrere Arbeiten publiziert worden, die sich mit Informationsverhalten [4] und der Bewertung von verfügbaren Patienteninformationen befasst haben [5, 6]. Auch das Infoportal Hautkrebs wird wissenschaftlich begleitet (AG Prof. F. Meier, Dresden und Prof. C. Berking, Erlangen).

Die Rückmeldungen durch die Teilnehmenden waren durchweg positiv. Die Evaluation zeigte als Hauptgründe das Erfahren von Selbstwirksamkeit, Wertschätzung individueller Themen, den Austausch un-

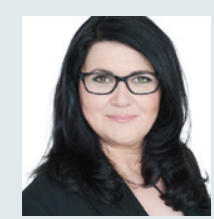

Kontaktadresse: Dr. Christiane Weber, MEDEA $\mathrm{GmbH}$, Medical Education Academy, Geschäftsführerin der MEDEA GmbH Medical Education Academy, Kommunikationstrainerin, Moderatorin, Dozentin, Freie Mitarbeiterin der ADO (Fortbildungen \& Kongresse), Mitglied der Arbeitsgruppe «Patientenkompetenz und Kommunikation» der NVKH, Winterberg 1, 66119 Saarbrücken, cweber@medea-health.de ter Gleichgesinnten sowie die gemeinsame Entwicklung neuer Ideen auf. Die Interaktion im virtuellen Raum lässt sich durch Tools für Umfragen, Online-Berichterstattung, Interviews oder Dokumentation der Sessions mit speziellen Programmen weiter ausbauen.

Das 2. Barcamp Hautkrebs findet voraussichtlich im März 2022 wieder als Präsenzveranstaltung statt. Vor allem die Teilnahme von DermatoOnkologen*innen wäre für das kommende Jahr wünschenswert.

Das Barcamp Hautkrebs 2020 fand mit freundlicher Unterstützung der Firmen Novartis, Roche und Sanofi Genzyme statt.

\section{Literatur}

1 Belliger A. Healthcare Transformation - von Systemen zu Netzwerken: Strategie- und Handlungsfelder zur Gestaltung der künftigen Arbeitswelt im Gesundheitswesen. In: Amelung V, Eble S, Sjuts R, et al., eds. Die Zukunft Der Arbeit Im Gesundheitswesen. MWV Medizinisch Wiss; 2020:57-66.Melanom Info Deutschland e.V. https://melanominfo.com/; Stand 2020.

2 Melanom Info Deutschland e.V. https://melanominfo.com/; Stand 2020

3 NVKH Report 2019/2020.https://nvkh.de/fileadmin/nvkh/downloads/NVKH_Report_2019-2020.pdf

4 Brütting J, Bergmann M, Garzarolli M, et al. Information-seeking and use of information resources among melanoma patients of German skin cancer centers. J Dtsch Dermatol Ges. 2018;16(9):1093-1101. doi:10.1111/ddg.13630

5 Brütting J, Steeb T, Reinhardt L, Berking C, Meier F. Exploring the Most Visible German Websites on Melanoma Immunotherapy: A Web-Based Analysis. JMIR Cancer. 2018;4(2):e10676. doi:10.2196/10676

6 Steeb T, Reinhardt L, Görgmayr C, et al. German YouTube TM videos a source of information on cutaneous melanoma: A critical appraisal. J Eur Acad Dermatology Venereol. April 2020. doi:10.1111/jdv.16510

\section{Aus- und Weiterbildung \\ Stellensuche neu gedacht}

Ärztinnen und Ärzte sind gefragt, die Situation am Arbeitsmarkt ist günstig. Paradoxerweise ist es für Ärztinnen und Ärzte dennoch schwierig, herauszufinden, an welcher Klinik die Arbeitsbedingungen den eigenen Anforderungen entsprechen. Die Macher der Online-Plattform Felixmedicus.de wollen daher der Stellensuche einen neuen Dreh verleihen und so den Weg zur Traumstelle erleichtern. Wie genau das funktionieren soll, darüber haben wir mit Niklas Wichmann, einem der Gründer von Felix Medicus, gesprochen.

\section{Felix Medicus hast du zusammen mit Leon Lüneborg und Thorben Schlätzer gegründet. Wie kam es zu der Idee?}

Niklas: Wir Ärztinnen und Ärzte machen während der Stellensuche häufig die Erfahrung, dass Medizinerinnen und Mediziner praktisch überall gesucht werden. Leider schlägt sich diese für uns günstige Arbeitsmarktsituation aber in vielen Kliniken nicht in besseren Arbeitsbedingungen nieder. In anderen Branchen mit Fachkräftemangel gibt es auf dem Arbeitsmarkt einen ausgeprägten Wettbewerb um gutes Personal. Damit einhergehend verbessern sich natürlich die Bedingungen für die Mitarbeiterinnen und Mitarbeiter. 
In der Medizin gibt es zwar auch Kliniken, die gute Arbeitsbedingungen bieten. Diesen fällt es jedoch häufig schwer, diese Vorteile gegenüber potenziellen Mitarbeiterinnen und Mitarbeitern klar zu kommunizieren.

Ärztinnen und Ärzte wissen natürlich durchaus, was ihnen in ihrer aktuellen Stelle fehlt, trauen sich aber nicht, das zu äußern oder aktiv nach einer neuen Stelle zu suchen. Und hier kommt Felix Medicus ins Spiel!

\section{Aus der Sicht einer Assistenzärztin, eines Assistenzarztes: Warum sollte ich mich bei Felix Medicus anmelden?}

Niklas: Felix Medicus gibt dir als Assistenzärztin oder Assistenzarzt die Möglichkeit, ein anonymisiertes Profil zu erstellen, indem du zunächst auflistest, welche Erfahrungen, Fähigkeiten und Fertigkeiten du bereits mitbringst. Außerdem kannst du deutlich machen, welche Vorstellungen du bezüglich der Arbeitsbedingungen und der fachlichen Weiterbildung an deine nächste Stelle hast. Wenn eine Klinik dir diese Vorstellungen erfüllen würde, kann sie dich anschreiben und ihr könnt ins Gespräch kommen.

Und warum sollten Kliniken oder Praxen euer Portal nutzen? Niklas: Für Kliniken und Praxen ist Felix Medicus eine einfache Möglichkeit, um Ärztinnen und Ärzte kennenzulernen, mit ihnen ins Gespräch zu kommen und deutlich zu machen, warum die eigene Klinik oder Praxis ein guter Ort für die Facharztweiterbildung ist. Die Kliniken, die bisher sehr einfach Personal finden, zeichnen sich ja in erster Linie durch eine gute geographische Lage aus. Wir wollen Kliniken und Praxen die Möglichkeit bieten, herauszustellen, was sonst noch für sie spricht - also etwa ein gutes Weiterbildungskonzept oder gute Arbeitsbedingungen. Durch eine offenere Kommunikation und daher einer treffsicheren Einstellung von neuen Mitarbeitern sinkt die Fluktuation und damit der Aufwand für die Kliniken.

\section{Ihr habt für Felix Medicus auch viele Assistenzärzte und} Assistenzärztinnen nach deren Wünschen, Hoffnungen und Pain-Points gefragt. Gibt es eine Art Top-5 der meistgenannten Punkte?

Niklas: Was das Fachliche angeht, wünschen sich viele Kolleginnen und Kollegen eine strukturierte Weiterbildung, die auch gelebt wird.

\footnotetext{
Was ist Felixmedicus.de?

Felix Medicus (www.felixmedicus.de) ist ein junges Startup aus Köln. Die Gründer (Leon Lüneborg, Thorben Schlätzer und Niklas Wichmann) bauen gerade eine Karriereplattform für Medizinerinnen und Mediziner auf. Auf dieser können Assistenzärztinnen und Assistenzärzte sich ein anonymisiertes Profil erstellen, in dem sie angeben, welche Erfahrungen und Fähigkeiten sie mitbringen und welche Wünsche und Anforderungen sie an ihren nächsten Arbeitgeber haben. Interessierte Kliniken oder Praxen, die diese Wünsche und Anforderungen erfüllen würden, können die Ärztinnen und Ärzte kontaktieren. So schaffen wir größtmögliche Transparenz. Dies führt für die Ärztinnen und Ärzte zu größerer Zufriedenheit und für die Kliniken zu geringerer Fluktuation.
}

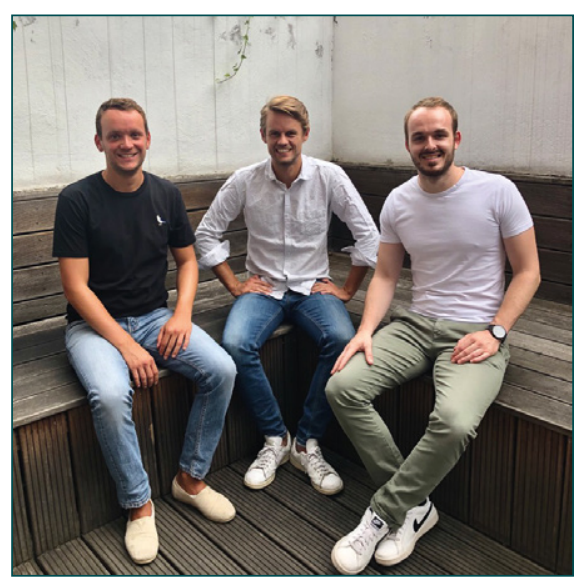

Die Gründer von Felix Medicus: Niklas Wichmann, Leon Lüneborg und Thorben Schlätzer

Außerdem ist eine gute Einarbeitung vor den ersten Diensten eine häufige Forderung der Ärztinnen und Ärzte - dies sollte ja eigentlich ohnehin eine Selbstverständlichkeit sein.

Auch bezüglich der Arbeitsbedingungen sind die Wünsche nicht utopisch. Viele wünschen sich verlässliche Arbeitszeiten oder einen Verzicht auf 24-Stunden Dienste. Teilweise wird aber auch nur Zeit für das Mittagessen gefordert.

Die Abhängigkeit in der Assistenzarztzeit von den Vorgesetzten ist groß. Da kann ein lautes Rufen nach besseren Arbeitsbedingungen oder ein angedeuteter Wechselgedanke schnell für dicke Luft und noch schlechtere Aussichten sorgen. Wie begegnet ihr dem Thema und schützt die Ärztinnen und Ärzte auf eurer Plattform?

Niklas: Die Profile bei Felix Medicus werden bewusst anonymisiert. Ein Rückschluss auf die eigene Person oder die aktuelle Klinik ist praktisch unmöglich. Die Ärztinnen und Ärzte laufen so keine Gefahr, dass der noch lose Wunsch nach einem Stellenwechsel zum Abteilungsgespräch wird.

\section{Zum Schluss noch die Rubrik «Wünsch dir was»: Wie stellst} du dir in Zukunft das Miteinander von Assistenzärztinnen, Assistenzärzten und Kliniken vor?

Niklas: Ich würde mir wünschen, dass Ärztinnen und Ärzte in der Weiterbildung wertschätzend und kollegial behandelt werden, dass eine echte Weiterbildung stattfindet und nicht nur die Stationsarbeit fertig werden muss. Ich glaube, dass sich uns Ärztinnen und Ärzten aktuell die Chance bietet, diese Wünsche auch zu realisieren. Der Arbeitsmarkt ist sehr günstig für uns und wir bekommen zum Beispiel durch die neue Weiterbildungsordnung mehr Flexibilität in der Gestaltung unserer Weiterbildung als zuvor.

Für die Zukunft bietet auch die Digitalisierung das Potenzial, dass ärztliche Arbeitszeit effizienter und sinnvoller genutzt wird, als dies bisher der Fall ist.

Ich würde mir wünschen, dass wir als Ärzteschaft diese großen Chancen gemeinsam in unserem Sinne gestalten.

Das Interview führte unser Redakteur Christoph Habel
214

Kompass Onkol 2020;7:210-216 DOI: $10.1159 / 000513212$ 
Aus- und Weiterbildung

Wie an der TU München digitale Kompetenz von Medizinstudierenden gefördert wird

Die Digitalisierung nimmt einen immer größer werdenden Stellenwert in der heutigen Gesellschaft ein und ist aus dem täglichen Leben nicht mehr wegzudenken.

Während in der freien Marktwirtschaft die Kommunikation und Arbeitsprozesse meist kontinuierlich dem neusten Stand der Digitalisierung angepasst werden, bestehen im medizinischen Bereich teilweise noch Probleme bei der Implementierung. Diese Hürden bestehen unter anderem dadurch, dass eine Umstellung auf digitale Prozesse in einem großen Krankenhaus mit über 100-jähriger Tradition und Bausubstanz mit einem enormen infrastrukturellen Aufwand und dementsprechenden Kosten verbunden ist oder es bezüglich hochsensibler Patientendaten ganz besondere Anforderungen an den Datenschutz sicherzustellen gilt. Durch diese Schwierigkeiten schreitet die Digitalisierung im Vergleich zu anderen wirtschaftlichen Sektoren gegenwärtig zwar vermeintlich nur langsam voran, ist jedoch nicht aufzuhalten. Aktuell gibt es eine Vielzahl an Beispielen für neue Technologien, die sich oft noch in der Entwicklungs- und Etablierungsphase befinden, die jedoch zukünftig einen großen Benefit für den Alltag des medizinischen Personals sowie Patienten und Patientinnen als auch der allgemeinen Bevölkerung bieten können.

\section{Idee zur Gründung der neuen Lehrveranstaltung}

Um die Digitalisierung in der Medizin weiter zu fördern, ist es wichtig, die Medizinstudierenden, Ärzte und Ärztinnen sowie alle im Gesundheitssektor tätigen Personen frühzeitig über die Möglichkeiten und das Potential von Digitalisierung aufzuklären. Aus diesem Grund wurde im Mai 2019 erstmalig die neu kreierte interdisziplinäre Lehrveranstaltung «Neue Technologie und die Krankenversorgung von morgen» unter der Leitung von PD Dr. Dr. Alexander Zink an der Fakultät für Medizin der Technischen Universität München angeboten. Seitdem wird die Veranstaltung in jedem Semester erfolgreich abgehalten, zuletzt unter dem neuen Namen «NIM: New Ideas for Medicine». Im Rahmen dieses zweitägigen Seminars sollen die Studierenden von internen und externen Referierenden unter anderem einen umfassenden Einblick darüber vermittelt bekommen, welche digitalen Technologien und Visionen es bereits gibt, welche digitalen Health Start-up Ideen gegenwärtig umgesetzt werden, wie künstliche Intelligenz (KI) funktioniert und wie diese beurteilt werden kann, welche Rolle das Internet in der heutigen Gesundheitsversorgung spielt und welche rechtlichen, soziokulturellen, ethischen und philosophischen Themen sich in diesem Kontext ergeben.

\section{Vor- und Nachteile des Internets als Informationsquelle}

Ziel des Seminars ist es ebenfalls den Studierenden einen Überblick darüber zu verschaffen, wie Digitalisierung bzw. digitale Daten für die Forschung genutzt werden können. Ein Schwerpunkt hierbei ist das Suchverhalten der Allgemeinbevölkerung auf Internetsuchmaschinen bezüglich gesundheitlicher Themen. Denn das Internet spielt eine immer bedeutendere Rolle im Bereich der «patient- journey». So zeigt die Arbeitsgruppe von Dr. Zink anhand von zahlreichen Publikationen zu dermatologischen Erkrankungen auf, dass das Suchvolumen in den letzten Jahren kontinuierlich zugenommen hat und es regionale Unterschiede in der Suchhäufigkeit sowie dem Suchverhalten gab. Dies kann auch zur Beantwortung medizinischer und versorgungswissenschaftlicher Fragestellungen herangezogen werden. Durch die qualitative Analyse identifizierter Suchbegriffe können zudem scheinbare «unmet medical needs» aufgedeckt werden, wie z.B. ein hohes Suchinteresse der deutschen Bevölkerung in Bezug auf Juckreiz im genitoanalen Bereich, was basierend auf klassischen Gesundheitsdaten bisher unerkannt blieb.

In der Lehrveranstaltung wird jedoch nicht nur hervorgehoben, wie Internetdaten zum Vorteil für die Forschung genutzt werden können, sondern auch welche Schwierigkeiten für die evidenzbasierte Medizin bestehen, dadurch, dass das Internet vermehrt für gesundheitliche Fragestellungen genutzt wird. Da den Nutzern und Nutzerinnen von Suchmaschinen oft tausende von vermeintlich relevanten Seiten vorgeschlagen werden, ist es für medizinische Laien extrem schwierig zu überblicken, ob die gefundenen Informationen auf Webseiten auf verlässlichen, wissenschaftlich belegten Quellen basieren.

Zudem werden von Betroffenen häufig Selbsthilfegruppen auf Social Media Plattformen wie Facebook genutzt, um sich mit anderen Betroffenen auszutauschen. Problematisch hierbei ist, dass immer wieder vermeintlich effektive Therapieoptionen empfohlen werden, die wissenschaftlich nicht belegt sind. Um diesen Problemen entgegen zu wirken, ist es von enormer Wichtigkeit, zum einen herauszufinden, welche Informationen ausgetauscht werden und zum anderen der Bevölkerung auf einfache Weise zu vermitteln, welche Informationen vertrauenswürdig sind.

\section{Natural language processing und Netzwerkanalysen}

Eine Methode die großen Datenmengen auf Social Media Plattformen zu analysieren ist «natuaral language processing» (NLP). Beim NLP wird eine KI genutzt, die die menschliche Sprache verstehen und interpretieren kann, wodurch Interessenschwerpunkte identifiziert werden können. Eine weitere Methode, um Big Data übersichtlich darzustellen, sind so genannte Netzwerkanalysen. Anhand von Netzwerkgraphen kann der Fokus in einem bestimmten Forschungsfeld basierend auf der Vernetzung von Wörtern, die beispielsweise in Publikationstiteln, Abstracts, Postern oder Blogs verwendet wurden, dargestellt werden. Eine Erweiterung zu einem interaktiven Tool, das die Daten mehrerer Jahre beinhaltet, ist dahingehend nützlich, dass die Entwicklung der Forschung sehr übersichtlich dargestellt ist. Das kann für die Planung zukünftiger Forschung sehr nützlich sein.

\section{Zusammenfassung}

Besonders wichtig ist den Veranstaltern und Veranstalterinnen die Interdisziplinarität des Seminars, um den Teilnehmern und 
Teilnehmerinnen die vielen Facetten der Digitalisierung aufzuzeigen. Entsprechend referieren und diskutieren in jedem Semester neben den Referierenden universitärer Medizin insbesondere auch Startup-Vertreter, große digitale Player, Politikvertreter und Politikvertreterinnen, wie zuletzt aus dem Bundesministerium für Gesundheit, Juristen und Juristinnen verschiedener Fakultäten und Schwerpunktfelder, Ethiker und Ethikerinnen der Hochschule für Philosophie München, Blockchain-Experten und Expertinnen aus der Finanzwelt, Cyber Security Experten und Expertinnen sowie Hacker und Hackerinnen.

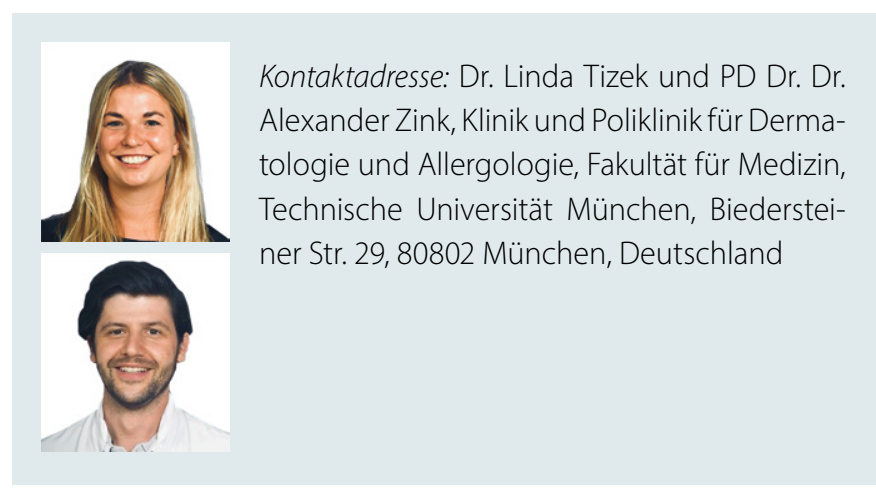

\section{Aus- und Weiterbildung \\ Der Umgang mit dem Pflegepersonal als Arzt}

Beginnt man seine Assistenz in einem Klinikum, trifft man oft auf sehr erfahrene Krankenschwestern und Krankenpfleger, die einem medizinische Tipps geben. Sollte man sie annehmen?

Um es vorwegzunehmen: Man kann die eingangs gestellte Frage nicht mit einem klaren «Ja» oder «Nein» beantworten, weil auch hier der Ton die Musik macht. Wenn eine erfahrene Schwester Vorschläge macht, was die Diagnose und Therapie von Patienten angeht, dann sollte man sich diese Vorschläge anhören. Pflegekräfte haben oft eine lange Berufserfahrung und die zunehmende Akademisierung ihrer Ausbildung macht den immer breiteren Erwerb von Fachwissen möglich. Dennoch bist du der Arzt oder die Ärztin und hast damit die Durchführungsverantwortung, während das Pflegepersonal in der Anordnungsverantwortung ist, das heißt du haftest später für die getroffene Entscheidung und nicht das Pflegepersonal. Wichtig ist deshalb, dass man Ratschläge anderer nicht vorschnell annimmt, sondern sich selbst in aller Ruhe ein Bild macht. Im Zweifel fragt man den Oberarzt oder den Weiterbildungsbevollmächtigten, ob die angedachte Therapie sinnvoll ist.

\section{Parallele Hierarchien}

Beim Pflegepersonal gibt es eine Pyramidenhierachie, die von der Hilfsschwester über die Schwester, zur Stationsschwester, zur Pflegedienstleitung bis zur Pflegedirektion reicht. Wie bei den Ärzten gibt es auch hier eine Weisungsbefugnis von oben nach unten. Grundsätzlich gilt: Die pflegerische Versorgung übernimmt das Pflegepersonal selbstständig, die medizinischen Aufgaben werden hingegen von dir als Arzt oder Ärztin delegiert und zwar sowohl an die Hilfsschwester, als auch an die Stationsschwester.

\section{Konflikte mit dem Pflegepersonal: 2 Beispiele}

Es ist oft gar nicht so leicht, als «Frischling» einer Stationsschwester Aufgaben zu erteilen. Deshalb ist mit großem Fingerspitzen- gefühl vorzugehen. Aber manchmal kann auch das größte diplomatische Geschick keine direkte Auseinandersetzung verhindern. Zur Verdeutlichung ein Beispiel:

Nehmen wir an, du beginnst deine Assistenz und die Stationsschwester, die erheblich älter ist, will von dir gesiezt werden, während sie dich mit «Du» anspricht. Auf sehr direkte Weise versucht sie also das Machtverhältnis umzukehren. Hier empfiehlt sich der direkte Weg der Kommunikation. Mach ihr deutlich, dass ihr euch in einem Dienstverhältnis befindet und du deshalb das höflichere «Sie» bevorzugst. Du wirst sie mit diesem Vorgehen nicht als Freundin gewinnen, aber sie hat es ja selbst auf die Konfrontation angelegt und ist damit eine schwierige Kollegin, mit der du ohnehin nicht warm geworden wärst.

Andererseits kann die Expertise des Fachpflegepersonals lebensentscheidend sein. In tragischer Weise verdeutlicht dies der Tod der Britin Elaine Bromiley. Sie verstarb aufgrund von Fehlern bei der Narkoseeinleitung, die teilweise vermeidbar gewesen wären, wenn die behandelnden Anästhesisten auf die Ratschläge der Anästhesieschwestern gehört hätten. Letztendlich geht es auf Station um Teamwork, bei der jeder sein Wissen einbringen dürfen sollte. Eine junge Ärztegeneration, die um ihre Verantwortung weiß, klare Grenzen zieht, aber auch gegenüber Kritik aufgeschlossen ist, kann in Zukunft sicherlich viel bewirken.

Duzt oder siezt man sich? Der Umgangston ist von Station zu Station unterschiedlich. spotmatikphoto/Fotolia 